\title{
“烯醛到芳醛”苯环化反应策略合成联芳基甲醛
}

\author{
谷 枫 姚伟军*
}

(浙江理工大学化学系 杭州 310018)

\section{"Enal to Aryl Aldehyde" Benzannulation Strategy for the Synthesis of Biaryl Aldehydes}

\author{
Gu, Feng Yao, Weijun* \\ (Department of Chemistry, Zhejiang Sci-Tech University, Hangzhou 310018)
}

芳醛类化合物是有机合成化学研究和相关应用中 不可替代的基本化学品, 其中具有联芳基骨架的联芳醛 类化合物不仅具有常规芳醛的性质, 更在很多前沿研究 领域, 如手性苂光探针合成、手性试剂制备以及多环芳 基稠环化合物构建等方面展示了重要而独特的应用. 已 有的联芳醛合成主要策略是官能团转化, 即联芳基底物 结构上的其它官能团向甲酰基的转化 ${ }^{[1]}$. 此外, 通过单 芳基醛和芳基偶联试剂之间的交叉偶联反应也是构建 联芳醛的常用方法 ${ }^{[2]}$. 特定情况下, 多步目标导向合成 的方法也可以实现某些联芳醛的合成 ${ }^{[3]}$. 对于已有的合 成策略而言, 需要采用联芳基化合物作为原料、过渡金 属催化和/或多步合成的效率问题仍在一定程度上限制 了相关合成方法的应用. 因此, 发展新的合成方法对已 有策略进行补充对于联芳醛类化合物仍然是重要研究 课题. 有趣的是, 尽管芳环化反应是当前合成芳香化合 物的主流策略之一, 对于含有甲酰基的芳醛而言, 这类 合成策略却长期以来难以得到应用. 主要原因是芳环化 反应通常需要较强的氧化条件来辅助芳构化过程, 而甲 酰基却对氧化条件敏感, 容易在芳环化的同时被氧化.

为解决这一问题, 江西师范大学化学化工学院万结 平课题组 ${ }^{[4]}$ 曾报道了烯胺酮和共轭二烯醛之间的 $[2+4]$ 型苯环化反应合成苯甲酫的方法. 这个方法的关键是在 环化反应之后, 通过氨基和 $\beta$-氢的消除, 在非氧化条件 下构建一个新的双键, 得到环己二烯结构的中间体. 因 为环已二烯在结构上具有更接近苯环的不饱和度, 因此 可以在极弱甚至无需氧化的条件下完成芳构化, 并不影 响体系中的醛官能团, 最终使得芳构化过程和醛官能团 在反应中实现了兼容, 揭示了通过非环状醛底物的苯环
化反应直接合成苯甲醛的可行性.

最近, 该小组 ${ }^{[5]}$ 基于烯胺消除模式下烯醛底物参与 的苯环化反应, 再次建立了联芳醛类化合物的直接合成 方法. 该方法通过丙炔酸酯加二级胺原位活化构建烯胺 酯的模式进行. 作者先以丙炔酸乙酯和肉桂酫的反应为 模型, 对反应条件进行了系统的优化, 建立了 2 equiv. 甲 胺 $\left(w=40 \%\right.$ 水溶液)为促进剂, 乙酸介质中 $80{ }^{\circ} \mathrm{C}$ 的反应 条件. 在该反应条件下，作者首先采用系列间位或对位 含有不同取代结构的肉桂醛和丙炔酸烷基酯反应, 顺利 得到了一系列含有不同取代基结构的联芳基-2-醛类化 合物 3, 产率中等到优秀(图 1A). 随后, 鉴于具有旋转 受阻轴不对称结构的芳醛类化合物的重要应用前景 ${ }^{[6]}$, 作者在扩展研究中采用了具有不同邻位取代的肉桂醛 作为底物和炔酸酯的反应, 对具有轴不对称结构的联芳 醛类化合物合成进行研究. 结果如图 1B: 在邻位含有 烷氧基、硝基和卤素取代基的产物都能顺利合成, 充分 展示了该方法的广泛适用性.

基于反应结果以及对潜在中间体的分离和相应控 制反应，作者提出了可能的反应机理(图 2). 反应主要经 历的可能中间体为 $5 、 6$ 和 7. 其中胺消除反应得到环己 二烯中间体 7 是该反应的关键步骤，环已二烯中间体形 成后, 可在弱氧化条件下实现芳构化, 在完成苯环构建 的同时不会影响甲酰基, 从而得到联芳醛类化合物.

总之, 万结平课题组发展了一条通过烯醛和炔酸酯 的苯环化反应合成联芳醛类化合物的新途径. 该方法可 通用于平面和具有轴不对称结构的联芳醛类化合物合 成. 此外，该方法不仅原料易得、操作简单和无需任何

* Corresponding author. E-mail: orgywj@zstu.edu.cn. Published online December 7, 2020. 


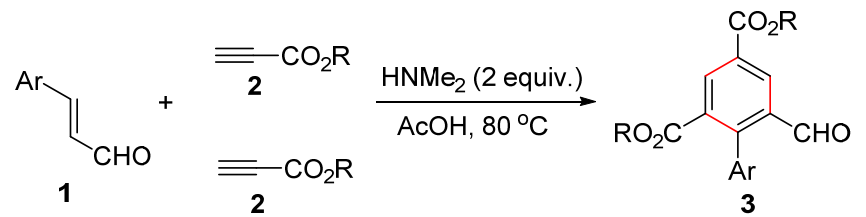

(A) Selected planar biaryl aldehydes

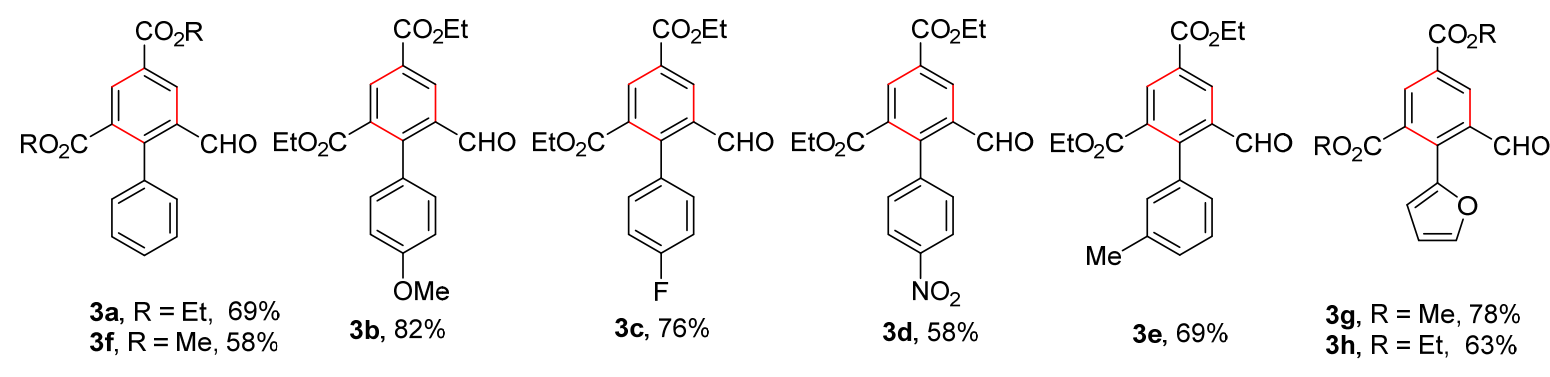

(B) Selected atropisomeric biaryl aldehyde

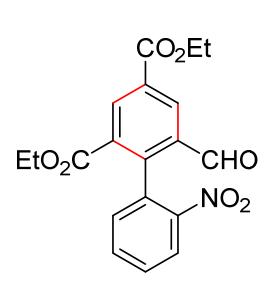

$30,56 \%$<smiles>CCOC(=O)c1cc(C)c(C(=O)OCC)c(-c2ccccc2Br)c1</smiles>

$3 v, 61 \%$<smiles>CC(=O)c1cc(C=O)c(-c2cccc3ccccc23)c(C(C)=O)c1</smiles>

$3 w, 52 \%$

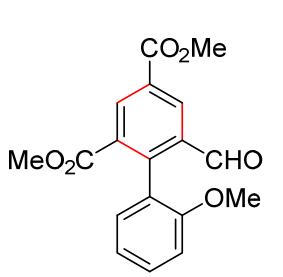

$3 p, 69 \%$

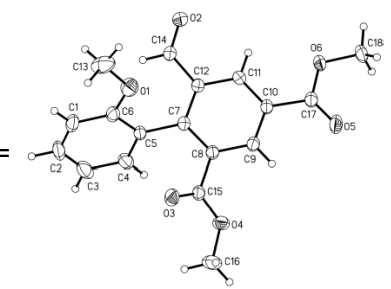

CCDC 2022967

图 1 基于烯醛苯环化反应合成联芳醛

Figure 1 Enal-based benzannulation for the synthesis of biaryl aldehydes

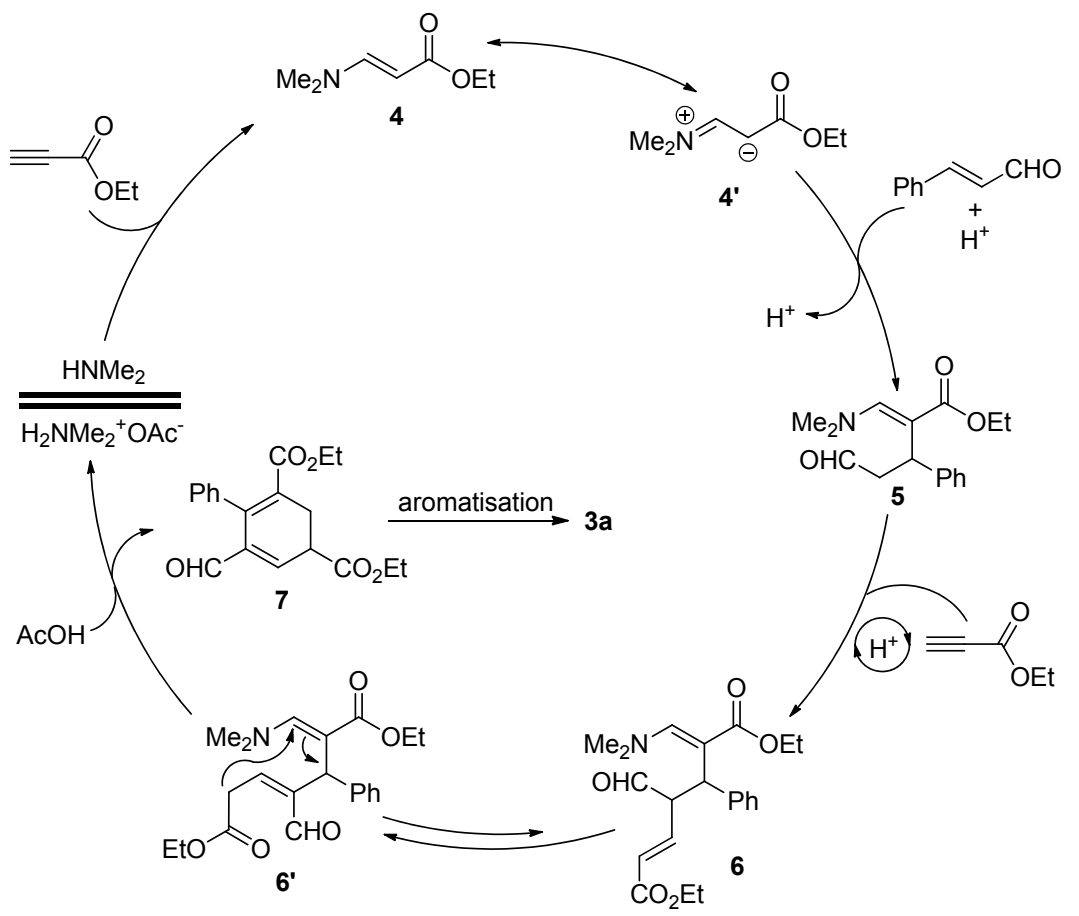

图 2 可能的反应机理

Figure 2 The plausible reaction mechanism

金属试剂，而且通过苯环化反应直接实现了从烯醛到联芳醛的合成，为芳醛合成提供了新的选择. 


\section{References}

[1] (a) Meyers, A. I.; Lutomski, K. A. J. Am. Chem. Soc. 1982, 104, 879.

(b) Schlosser, M.; Bailly, F. J. Am. Chem. Soc. 2006, 128, 16042.

[2] (a) Song, H.-J.; Jiang, W.-T.; Zhou, Q.-L.; Xu, M.-Y.; Xiao, B. ACS Catal. 2018, 8, 9287.

(b) Ding, L.; Sui X.; Gu, Z. ACS Catal. 2018, 8, 5630.

[3] Link, A.; Sparr, C. Angew. Chem., Int. Ed. 2014, 53, 5458.
4] Yang, L.; Wan, J.-P. Chem. Commun. 2018, 54, 7475.

[5] Hu, D.; Yang, L.; Wan, J.-P. Green Chem. 2020, 22, 6773.

[6] (a) Feng, J.; Gu. Z. Chin. J. Org. Chem. 2020, 40, 1787 (in Chinese).

(冯佳, 顾振华, 有机化学, 2020, 40, 1787.)

(b) Zhu, Y.-Y.; Wu, X.-D.; Gu, S.-X.; Pu, L. J. Am. Chem. Soc. 2019, 141, 175.

(c) Li, S.; Chen X.-Y.; Enders, D. Chem 2018, 4, 2026.

(Cheng, F.) 